\title{
Sorafenib from palliative to neoadjuvant chemotherapy in hepatocellular carcinoma with major vascular invasion: experience of two cases
}

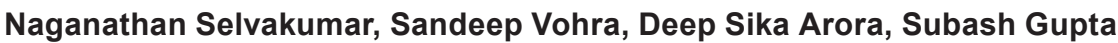 \\ Indraprasta Apollo Hospital, Sarita Vihar, New Delhi 110074, India
}

Correspondence to: Dr. Naganathan Selvakumar, Liver Transplantation Surgery, Indraprasta Apollo Hospital, Sarita Vihar, New Delhi 110074, India. E-mail: enselva1@gmail.com

How to cite this article: Selvakumar N, Vohra S, Arora DS, Gupta S. Sorafenib from palliative to neoadjuvant chemotherapy in hepatocellular carcinoma with major vascular invasion: experience of two cases. Hepatoma Res 2017;3:18-21.

\author{
Article history: \\ Received: 12-05-2016 \\ Accepted: 14-11-2016 \\ Published: 12-01-2017

\section{Key words:} \\ Hepatocellular carcinoma, \\ sorafenib, \\ hepatic artery ligation, \\ liver transplantation, \\ major vascular invasion
}

\section{ABSTRACT}

Hepatocellular carcinoma (HCC) is the fifth most common malignancy worldwide and the incidence is higher in cirrhosis. Treatment options depend on tumor stage, status of liver function, and the general condition of the patient. Major vascular invasion is a contraindication for liver transplantation. Sorafenib has been found to be useful in association with transarterial chemoembolization as an effective chemotherapeutic agent to prolong survival in inoperable HCCs. Here we describe our experience where sorafenib was used as palliation but later turned out to be a neoadjuvant. Both cases had major portal vein thrombosis and received sorafenib as palliative therapy. After a mean use of 6 months, both patients had marked tumor response and proceeded to have liver transplantations. Both cases are tumor-free at a median follow up of 13 months.

\section{INTRODUCTION}

Hepatocellular carcinomas (HCCs) are the fifth most common cancers in the world. The incidence of HCC is more in the eastern population compared to the west. Incidence is also higher in the cirrhotic livers as compared to the non cirrhotics. Management depends on the tumor stage, status of the liver and general physical status of the patient. Majority of HCC patients at the time of primary consultation have advanced and incurable. Hence there are many palliative options available to prolong the survival in such group of patients. In patients with early cancers curative treatment options are possible. Curative options include liver resection, liver transplantation, radiofrequency ablation (RFA). Palliative therapeutic options include transarterial chemoembolization (TACE), transarterial radioembolisation, sorafenib, external beam radiotherapy (EBRT), combination chemotherapy regimens. With recent advances in

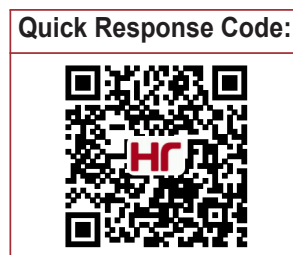


liver transplantation various neoadjuvant modalities have evolved over years to make inoperable patients into operable with equivalent survival rates. TACE, RFA and EBRT have been employed as neoadjuvant modalities to reduce the tumor burden. There are resolution chest tomographies (RCTs) going on to assess the effect of neoadjuvant role of TACE with or without sorafenib. Our case reports give a different perspective to these ongoing studies. One case was sorafenib without hepatic artery occlusion and the other one with hepatic artery occlusion.

\section{CASE REPORT}

\section{Case 1}

A 54-year-old gentleman, a business man from Islamabad, was diagnosed with hepatitis $C$ virus (HCV) infection in 2003 when he was worked up for generalized weakness. For which he received 26 injections of peg-interferon over 3 months and achieved sustained viral response (SVR). He remained relatively asymptomatic till 2015 . In September 2015, he developed right upper quadrant pain associated with significant loss of weight. In October 2015, he was diagnosed with HCC in the right lobe with portal vein tumor thrombosis (PVTT) and encasement of right hepatic vein and middle hepatic vein. The alpha fetal protein (AFP) levels rapidly increased to $>50,000$ by November 2015. In view of the advanced nature of the disease, he was started on sorafenib $400 \mathrm{mg}$ twice daily in Pakistan. He was reevaluated in our institute and found out to be not a candidate for liver transplantation. Since the cirrhosis was of Child A status, and imaging showed adequate remnant (there was right portal vein thrombosis causing adequate hypertrophy of the left lobe), he was subjected to exploratory laparotomy with the intention of palliative tumor resection on November 24, 2015. But at laparotomy, there was a large mass arising from the right liver with adherence to the colon. There were no signs of any distal metastasis. So the surgery was concluded after doing right hepatic artery ligation. His post procedure period was uneventful and was discharged on November 28, 2015. Tab sorafenib $400 \mathrm{mg}$ bid was continued post operatively. In the second week of April 2016, he developed cutaneous manifestation of drug intolerance, hence discontinued. During this period, the AFP level in January 2016 had decreased to 1,303 and the patient had shown improvement in his general condition. A positron emission tomography-computed tomography (PET-CT) was repeated in April 2016 which showed features of tumor necrosis and bland PVT without any evidence of distant metastasis. His AFP had dramatically decreased to $3 \mathrm{IU} / \mathrm{mL}$ [Figure 1]. As he did not have any radiological signs of viable disease the plan for palliative radiotherapy was cancelled. After assessment for living-donor liver transplantation (LDLT) and after discussion of the case in the liver transplant meeting, it was decided to do LDLT.

On admission, investigations revealed $\mathrm{Hb}$ 12.10, TLC $5,860 / \mathrm{cu} \mathrm{mm}$, platelet count 198,000/cu mm, prothrombin time/international normalized ratio (PT/INR) 9.40/0.90, urea $25 \mathrm{mg} / \mathrm{dL}$, creatinine $0.70 \mathrm{mg} / \mathrm{dL}$, serum bilirubin $0.60 \mathrm{mg} / \mathrm{dL}$, albumin $3.60 \mathrm{mg} / \mathrm{dL}$. Anti HCV was reactive and HBsAg \& HIV were non-reactive. Serum AFP was $3.52 \mathrm{IU} / \mathrm{mL}$. Urine protein/creatinine ratio was 0.24 . PET-CT liver showed cirrhotic liver with a small right lobe and multiple SOL's in the residual right lobe and tumor thrombus in right portal veins and main portal veins/left portal veins junction as described, mild ascites. Magnetic resonance imaging upper abdomen showed liver cirrhosis, multiple masses in both lobes of liver (right > left) with tumor thrombus in right, left and main portal vein near portal bifurcation suggestive of HCC, bland thrombus in remaining portal vein, no significant abdominal lymphadenopathy or ascites is seen. High RCT showed no scan evidence of pulmonary metastasis. 2D Echo showed pulmonary artery systolic pressure 22, CVP 5, EF $60 \%$ and dobutamine stress echocardiography was negative. Considering the nature of disease and explaining the risk/prognosis to relatives, he was planned for liver transplantation. After optimization and PAC clearance, patient was taken up for surgery on April 21, 2016.

He received a modified right lobe graft with graft recipient weight ratio of $>1$ on April 21, 2016. Post operatively he was shifted to the intensive care unit and was extubated on post operative day (POD) 1 according to the protocol. Immunosuppressant were started on POD 1 according to the protocol. Patient was started on liquid diet on POD 2 and gradually increased to normal diet. His lab reports showed a steady improvement with a peak bilirubin of 2.8 and a peak INR of 2.9 on POD 1. His both drains were removed on

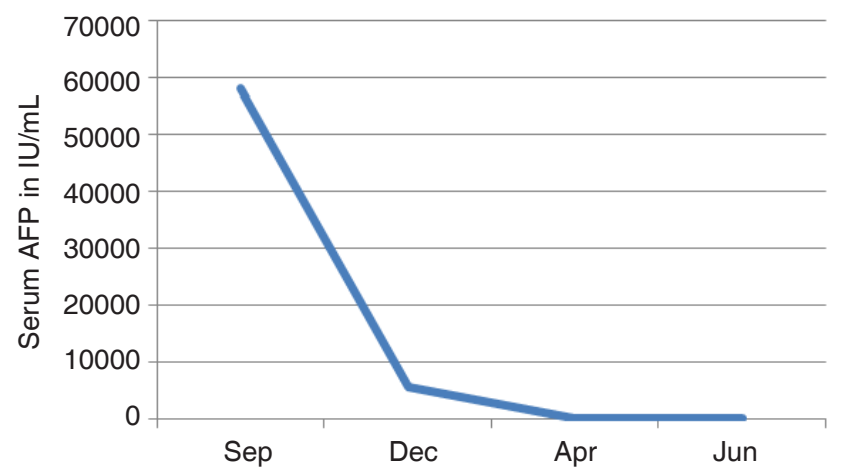

Figure 1: Alpha fetal protein (AFP) trend of case 1 


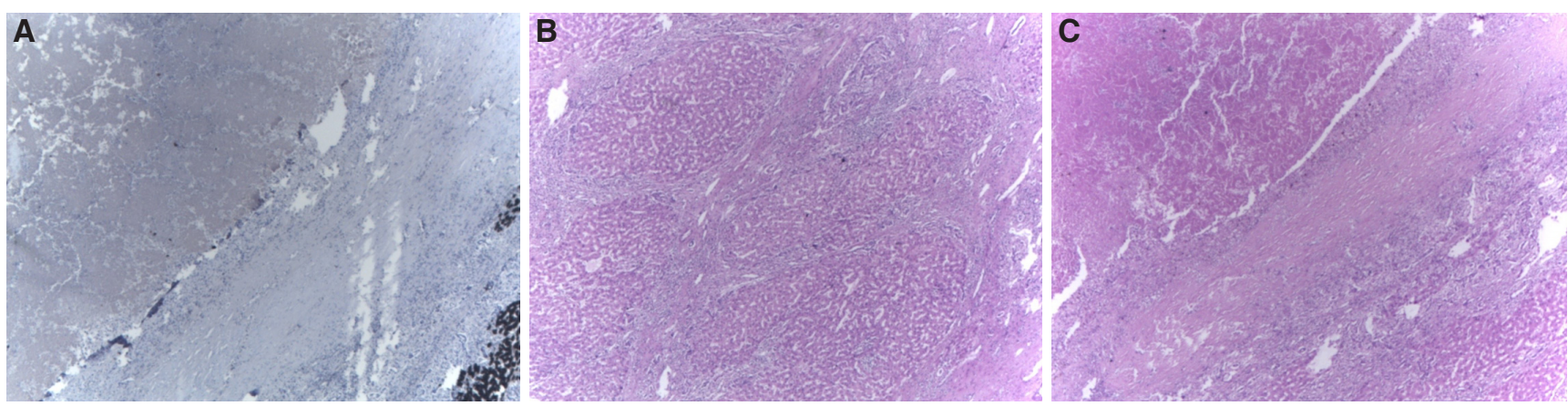

Figure 2: The final istopathology results of case 1. (A) AFP stain showing necrotic tumor, $\times 4$; (B) back ground cirrhosis $(\mathrm{HE}, \times 4)$; (C) necrotic tumor $(\mathrm{HE}, \times 10)$

POD 6. He tolerated immunosuppression well. He was discharged in a stable condition on POD 13.

The final histopathology of the explant specimen did not show any tumor at all. There was complete tumor response to hepatic artery ligation and sorafenib therapy [Figure 2].

At 14 months post transplantation, he has been switched over to everolimus based immunosuppression. Also he is on adjuvant sorafenib treatment. At 13 months post transplantation his serum AFP is normal and PET-CT is normal. Graft functions are normal.

\section{Case 2}

A 48-year-old gentleman from Sindh Pakistan was a case of HCV related chronic liver disease. He was diagnosed in 2012 with HCV. He received interferon therapy and achieved SVR. In June 2013 he was diagnosed with HCC and PVT along with elevated AFP. He was given sorafenib treatment. Subsequent follow up revealed normalization of AFP, clearance of PVTT and decrease in the tumor size. Sorafenib therapy was discontinued after 4 months owing to intolerance. He was on regular follow up with 3 monthly AFP and CT scan. The AFP was normal and the tumor was more or less constant size of $4.5 \mathrm{~cm}$ with no evidence of new lesions elsewhere. In view of the PVTT in previous scans, transplantation was deferred by various transplantation centers. However, in June 2015 he developed severe encephalopathy followed by recurrent episodes of minor encephalopathies. In view of hepatic decompensation, he underwent liver transplantation in October 2015. Post transplantation explant biopsy revealed low grade HCC in Milan with no capsular or vascular invasion. He had uneventful post-operative course. At 14 months post transplantation, patient survival and graft survival are good with no tumor recurrence.

\section{DISCUSSION}

HCCs are the commonest primary neoplasms of the liver. They are the fifth most common cancers with 4th commonest malignancy. There are multiple etiologies for HCCs. In general, cirrhotic livers have higher incidence of HCC as compared to non cirrhotics. The duration of cirrhosis is directly proportional to the cumulative incidence of malignancy. HCC has peculiar tumor biology. Curative treatment options for HCC are RFA, resection and liver transplantation. ${ }^{[1]}$ Of these three, primary liver transplantation has better survival in patients with cirrhosis and HCC. ${ }^{[2]}$ The indications for liver transplantation in CLD with HCC has been gradually expanding since the publication of Milan criteria. It started from Milan criteria and has reached to any size any number without vascular invasion criteria. ${ }^{[3]}$ Even in cases of vascular invasion there are case series to prove the efficacy of neoadjuvant radiotherapy (branchy/EBRT) with reasonable recurrence free survival rate. ${ }^{[4]}$ In case 1 where the intention was purely palliative but later on patient ended up with successful liver transplantation. Initial look up of the case was suggestive of hopeless situation. Hence we abandoned the resection attempt after ligation of the hepatic artery. There was no decompensation in the post-operative period. $\mathrm{He}$ received sorafenib as palliative chemotherapy protocol. Decision making for liver transplantation was crucial in this case. However, we went by basic tumor assessment methods like serum AFP, PET avidity and contrast enhancement of tumor and thrombus. Since all three parameters were negative he was taken up for transplantation. There are trials which showed improved survival in HCC patients who had received TACE+ sorafenib instead of either one alone. However, there is no case report so far in the literature where a patient with massive portal vein tumor thrombus has had complete tumor response after hepatic artery blockage and sorafenib therapy. We do not know whether the response was purely to Hepatic artery ligation or it is cumulative response to sorafenib also. ${ }^{[5]}$

The case 2 we described received sorafenib with palliative intent. But follow-up evaluation with CT 
scan and serum AFP revealed good tumor response in the form of clearing of portal vein thrombosis and reduction of AFP.

These two cases give us additional hope that PVT is not the end of the story in HCC patients. Though today the standard of care for HCC with PVTT is EBRT followed by reassessment and transplantation once tumor thrombus clears. ${ }^{[6]}$ We believe that sorafenib plays definite role as a neoadjuvant therapy.

In conclusion, high AFP and major vascular invasion should not be considered as end points in treatment of HCC patients. Neoadjuvant modalities are to be employed followed by reevaluation for transplantation. Since final conclusion needs high experience with more number of cases individual discretion is advised before offering transplantation in these patients.

\section{Financial support and sponsorship}

None.

\section{Conflicts of interest}

There are no conflicts of interest.

\section{Patient consent}

Patient consent is not needed as there is no disclosure of any personal information of patients.

\section{Ethics approval}

This is not needed as it is not an experimental study.

\section{REFERENCES}

1. Marrero JA, Welling T. Modern diagnosis and management of hepatocellular carcinoma. Clin Liver Dis 2009;13:233-47.

2. Bhangui P, Allard MA, Vibert E, Cherqui D, Pelletier G, Cunha AS, Guettier C, Vallee JC, Saliba F, Bismuth H, Samuel D, Castaing D, Adam R. Salvage versus primary liver transplantation for early hepatocellular carcinoma: do both strategies yield similar outcomes? Ann Surg 2016;264:155-63.

3. Asham EH, Boktour M, Ghobrial RM. Liver transplantation for hepatocellular carcinoma: past, present, and future. Clin Transpl 2012:173-83.

4. Yao FY, Kinkhabwala M, LaBerge JM, Bass NM, Brown R Jr, Kerlan R, Venook A, Ascher NL, Emond JC, Roberts JP. The impact of pre-operative loco-regional therapy on outcome after liver transplantation for hepatocellular carcinoma. Am J Transplant 2005;5:795-804

5. Vitale A, Volk ML, Pastorelli D, Lonardi S, Farinati F, Burra P, Angeli P, Cillo U. Use of sorafenib in patients with hepatocellular carcinoma before liver transplantation: a cost-benefit analysis while awaiting data on sorafenib safety. Hepatology 2010;51:165-73.

6. Lee DS, Seong J. Radiotherapeutics options for hepatocellular carcinoma with portal vein tumor thrombosis. Liver Cancer 2014;3:18-30. 